8th Alexander Friedmann International Seminar

on Gravitation and Cosmology

International Journal of Modern Physics: Conference Series

No World Scientific

Vol. 3 (2011) 507-514

(C) World Scientific Publishing Company

DOI: $10.1142 / \mathrm{S} 2010194511001024$

\title{
MEASUREMENT OF THE CASIMIR INTERACTION BETWEEN A Au SPHERE AND Au GRATINGS
}

\author{
RICARDO S. DECCA \\ Department of Physics, Indiana University-Purdue University Indianapolis, \\ Indianapolis, IN 46202, USA \\ rdecca@iupui.edu
}

Received 30 June 2011

Revised 8 July 2011

\begin{abstract}
The measurements between a Au coated sphere and Au gratings with different structural parameters are presented. While the main effect of the grating is to reduce the interaction observed between the same sphere and a $\mathrm{Au}$ plane at the same distance, it is observed that the reduction is non-trivial. While at small separations the interaction is greater than what is expected from purely geometrical effects, at large separations the effect not only becomes smaller but it also presents a faster decay than the corresponding geometrical contribution.
\end{abstract}

Keywords: Casimir force modification; boundary conditions.

PACS numbers: 12.20.Fv, 03.70.+k, 12.20.Ds, 42.50.Lc

\section{Introduction}

Since H. B. G. Casimir ${ }^{1}$ first predicted the attractive force existing between neutral perfectly conducting plates, a significant amount of research has been performed. Most of these investigations were fuelled by better measurements of the interaction. ${ }^{2-11}$ These improved measurements required the use of more sophisticated models ${ }^{12-15}$ to take into account finite conductivity effects, finite temperature, roughness, and also different geometries. So far, a complete picture that includes all previous effects is lacking. It is clear, however, that the effect is the result of the complicated interplay between the allowed modes in the cavity defined by the neutral bodies.

Experimentally, different approaches have been used to control the interaction induced by zero point fluctuations in the cavity. Changes in the electromagnetic response of the neutral materials, ${ }^{16,17}$ the properties of the dielectric between the neutral bodies, ${ }^{18}$ and even the geometry have been explored. ${ }^{19-21}$ Chiu and cowork$\mathrm{ers}^{21}$ showed that the effect of a sinusoidal corrugation in the surfaces is not properly described by the proximity force approximation.

In the work performed by Chan's group, ${ }^{20}$ the effect of a grating in one of the bodies has been investigated. It has been observed that the results are not trivial 
and cannot be explained by a pairwise addition approximation or the proximity force approximation.

In our present work the effect of a grating in one of the surfaces is investigated. Differently from Chan's group work, ${ }^{20}$ both surfaces are made out of Au. Also, contrary to what was done in corrugated Au surfaces, ${ }^{19,21}$ the studied gratings have a large aspect ratio.

\section{Experimental Setup}

The experimental setup used during this project is similar to the one used in our previous works that allowed us to produce the most precise measurement of the Casimir force between metallic surfaces with a sensitivity of $6 \mathrm{fN} /(\mathrm{Hz}) 1 / 2 .{ }^{11}$ The schematic of the system used can be seen in Fig. 1. It consists of a metal-coated sphere with radius $R \sim 150 \mu \mathrm{m}$ attached to a microelectromechanical (MEMS) torsional oscillator. The metallic nanostructured surface (metallic grating) is attached to an optical fiber that is used to measure distances very precisely. As the metallic grating is brought into close proximity of the gold-coated sphere, the interaction between the two metallic surfaces produces both a rotation in the oscillator and a change in its resonance frequency. Both these quantities are measured in our experiments and used to extract the Casimir force between the nanostructure surface and the metallic sphere. As in all determinations of the Casimir interaction, the measurements are divided into three main steps: (i) Calibration of the MEMS system; (ii) Separation measurement between the two surfaces involved; and (iii) measurement of the interaction itself.

All the samples fabricated using the process described in Sec. 3 have the layout shown in Fig. 2. In these samples, the nanostructured surface, or nanoscale grating, is limited on two sides by a continuous metallic film. This layout allows direct comparison between the Casimir force measured in the continuous and the

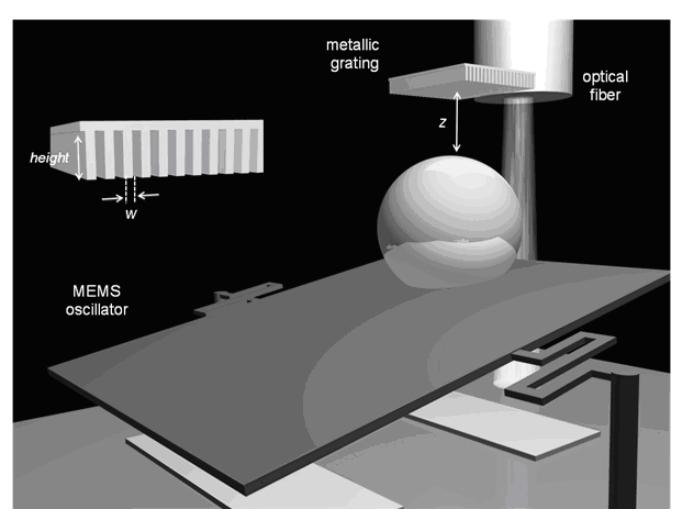

Fig. 1. Experimental setup used to measure the Casimir force between a metallic nanoscale grating and a gold coated sphere. 

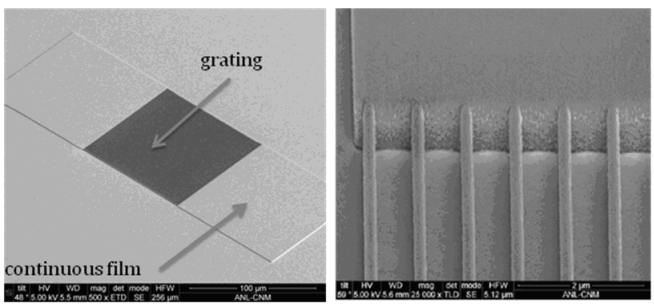

Fig. 2. SEM picture of a sample used to detect the Casimir interaction. The central part of the sample is the nanostructured region and the lateral areas consist of continuous films without any nanostructure. The right panel shows a detail of the area where the grating merges the continuous film.

nanostructured section of the samples, avoiding the concerns associated with variation of materials properties due to fabrication reproducibility.

In the electrostatic calibration, ${ }^{22}$ the sphere is placed on top of the continuous part of the sample. The observed variations in the resonance frequency of the oscillator as $z$ is varied are compared with the expected values from the interaction between a sphere and a plane. These electrostatic calibrations are also used to obtain the sensitivity of the oscillator. While performing Casimir interaction experiments, a potential difference between the sphere and the plate is applied, nullifying the electrostatic interaction. Unlike the static regime where forces are measured, in the dynamic regime the force gradient $\partial F / \partial z$ is measured by observing the change in the resonant frequency of the MEMS oscillator as the sphere-grating separation changes. Using the change of the resonance frequency of the oscillator and the gradient of the Casimir force, ${ }^{23}$ the equivalent Casimir pressure $\left(P_{C}\right)$ between two infinite plates of the same materials separated by $z$ can be obtained. A typical experimental

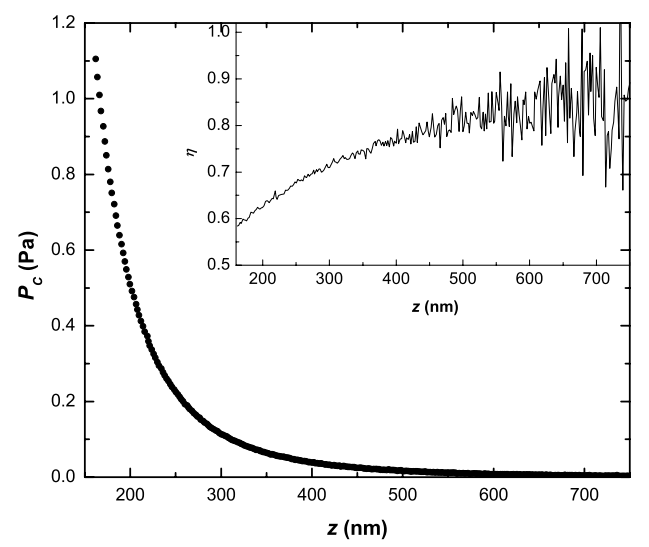

Fig. 3. Equivalent Casimir pressure determined for a flat sample. The inset shows the ratio $\eta=P_{C} / P_{\text {ideal }}$. 
Table 1. Parameters of all the nanostructured Au samples measured.

\begin{tabular}{rlrrr}
\hline Sample & Fabrication & $\begin{array}{c}\mathrm{h} \\
(\mathrm{nm})\end{array}$ & $\begin{array}{c}\mathrm{w} \\
(\mathrm{nm})\end{array}$ & $\begin{array}{c}\mathrm{p} \\
(\mathrm{nm})\end{array}$ \\
\hline 1 & Electroplating & 214 & 88 & 250 \\
2 & Electroplating & 214 & 99 & 250 \\
3 & Electroplating & 214 & 116 & 300 \\
4 & Electroplating & 216 & 90 & 250 \\
5 & Electroplating & 216 & 92 & 250 \\
6 & Electroplating & 280 & 100 & 300 \\
7 & Electroplating & 400 & 130 & 350 \\
8 & Electroplating & 420 & 120 & 500 \\
9 & coated HSQ & 400 & 130 & 300 \\
10 & coated HSQ & 400 & 150 & 400 \\
11 & coated HSQ & 400 & 170 & 400 \\
12 & coated HSQ & 400 & 110 & 600 \\
13 & coated HSQ & 400 & 130 & 600 \\
14 & coated HSQ & 400 & 190 & 600 \\
15 & coated HSQ & 400 & 210 & 600 \\
16 & coated HSQ & 400 & 150 & 800 \\
17 & coated HSQ & 400 & 170 & 800 \\
\hline
\end{tabular}

result obtained for a continuous film is shown in Fig. 3. The inset of Fig. 3 shows the same data but normalized by the Casimir interaction between perfect plates made of an ideal metal,

$$
P_{\text {ideal }}(z)=\frac{\hbar c \pi^{2}}{240 z^{4}}
$$

\section{Samples}

Several nanostructured surfaces with different pitch $p$, ridge width $w$ and height $h$ have been measured (see Table 1). The samples have beed grown by two different methods, coating of HSQ photoresist and electroplating.

(i) Au coated HSQ gratings: In this nanofabrication method high aspect ratio metallic nanostructures are created with Au coating of HSQ (hydrogen silsesquioxane). HSQ is an inorganic negative tone e-beam resist which is basically a spin-on dielectric with silicon dioxide-like physical properties. HSQ can be patterned with e-beam lithography down to $6 \mathrm{~nm}$ feature sizes and be cured into a robust dielectric material. $380 \mathrm{~nm}$ thick and $40 \mathrm{~nm}$ wide lines can be patterned to create 10:1 aspect ratio structures. After patterning, these HSQ structures are coated with Au by conformal sputter deposition. The conformality of our deposition is around 0.25 and hence the widening of the lines is minimal but thick enough to completely cover the dielectric with $\mathrm{Au}$. Hence, a $40 \mathrm{~nm}$ wide line becomes a $\sim 100 \mathrm{~nm}$ wide line after coating with $\sim 130 \mathrm{~nm}$ of metal. This results in $100 \mathrm{~nm}$ wide and $\sim 500 \mathrm{~nm}$ tall 5:1 aspect ratio structures. This method does have its limitations in the smallest width achievable due to the minimal thickness $(\sim 70 \mathrm{~nm})$ required for $\mathrm{Au}$ in 
Casimir measurements. HSQ itself has very smooth surfaces after patterning and hence result in a smooth $\mathrm{Au}$ surface when coated. The Au surface quality from AFM measurements show that the deposited Au has a RMS surface roughness of about $1 \mathrm{~nm}$ and is comparable to the surface roughness of $\mathrm{Au}$ deposited on single crystal silicon.

(ii) Electroplated gratings For the fabrication of electroplated nanostructures the following process was used. ZEP positive e-beam resist with thickness of 500 $\mathrm{nm}$ was spun on a gold seed layer. The resist was exposed at $100 \mathrm{kV}$ to form a high-resolution, high-aspect ratio mold. Gold was plated from a cyanide solution to varying thicknesses, and then the resist was stripped in solvent. The measured RMS roughness of the lines is on the order of $1.5 \mathrm{~nm}$, and the thickness variation across a structure is approximately $10 \mathrm{~nm}$. The sample with narrowest lines obtained with this technique, has the following dimensions: $w=80 \mathrm{~nm} ; p=250 \mathrm{~nm} ; h=220 \mathrm{~nm}$

\section{Results}

Results obtained in nanostructured samples having similar filing factor $f=w / p$ (samples 1 to 6 in Table 1) are described in this work. To have a good characterization of the separation between the sphere and the grating, the height $h$ of the gratings was determined by means of an atomic force microscope. It was checked that experimentally indistinguishable results were obtained if the whole electrostatic calibration process was done over the grating itself (in this case, the capacitance between the sphere and the grating was calculated by solving the electrostatic problem using finite elements).

The measured Casimir pressures between the sphere and the different nanostructured surfaces are shown in Fig. 4. For comparison, the Casimir pressure between the sphere and a continuous film is also shown (dotted line). A clear reduction of the

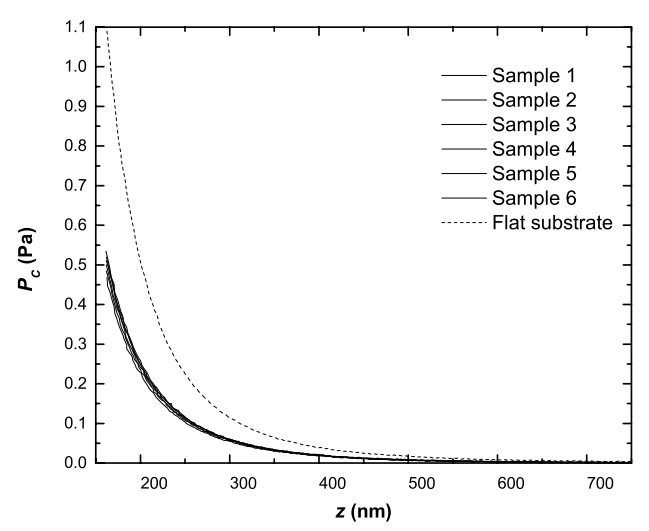

Fig. 4. Experimental Casimir pressure for six nanostructured samples with similar filling factor. The dotted line is the experimental result obtained for a continuous flat film. 


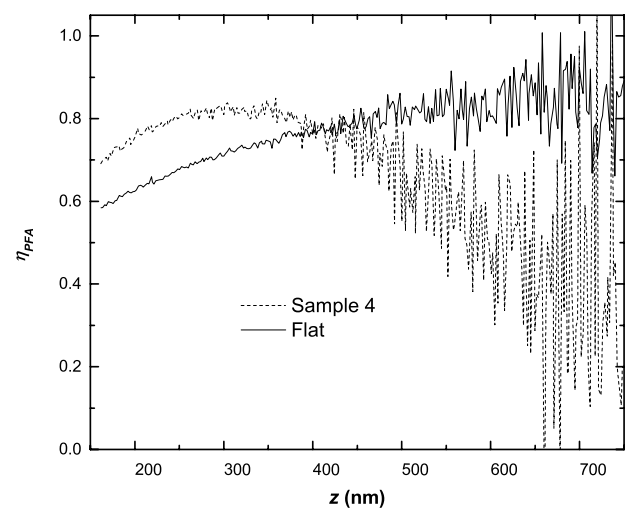

Fig. 5. Normalized Casimir interaction for a nanostructured sample (dotted line) and a continuous flat film of gold (full line). A clear crossover is seen at distances $\sim 400 \mathrm{~nm}$.

Casimir interaction is observed when comparing to the planar case. As expected, all the nanostructured samples present a similar Casimir pressure since all of them have very similar filling factor (see Table 1).

Totally unexpected results appear when the Casimir pressure per unit of area is calculated for these samples. Figure 5 shows the same data of Fig. 4 but normalized by a geometrical factor. In Fig. 5

$$
\eta_{P F A}(z)=\frac{P_{\text {grat }}(z)}{f P_{\text {ideal }}(z)+(1-f) P_{\text {ideal }}(z+h)}
$$

is plotted. In Eq. (2), $P_{\text {grat }}(z)$ is the measured Casimir interaction between the sphere and the grating, separated by a distance $z$, as plotted in Fig. 4 . For the particular case of a continuous sample, $w=p$ and $\eta_{P F A}$ reduces to $\eta$.

The data clearly shows that at small separations the nanostructured surfaces have a more attractive interaction per unit area than the flat surface. At larger separations the situation is reversed: the Casimir interaction per unit area is less attractive than in the case of a flat substrate. Figure 5 shows only the results of sample 4 but this behavior has been observed in all the measured samples. Furthermore, a clear trend has been observed when comparing data of nanostructured samples with same filling factor but different period: the smaller the period of the grating, the larger the reduction of the Casimir force (see Fig. 6).

This is the most important result of our work. It clearly demonstrates that by patterning the surface of the interacting objects with nanoscale features the intensity of the attractive Casimir interaction can be reduced. The magnitude and length scale of the force can be controlled by the specific details of the pattern etched on the interacting surfaces. It is worth observing that the long separations decay of $P_{\text {grat }}$ is faster than $z^{-4}$. 


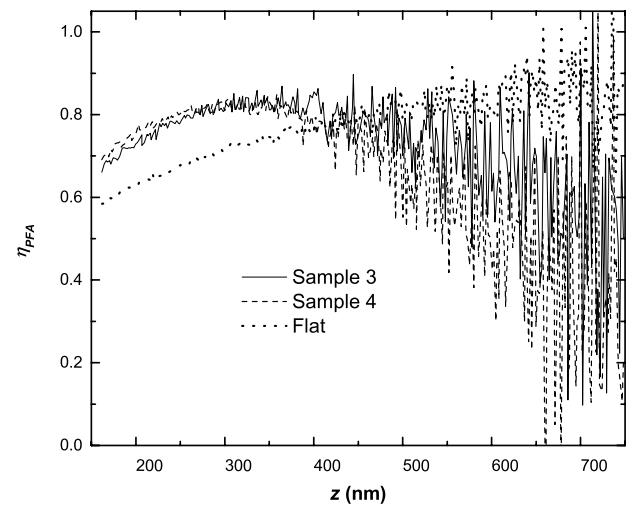

Fig. 6. Normalized Casimir interaction for two different nanostructured surfaces with similar filling factor and different pitch (full and dashed lines). For comparison the Casimir pressure of a continuous flat substrate (dotted line) is included.

\section{Conclusions}

It has been shown in this work that the intensity of the Casimir force in vacuum in a real experimental system can be controlled by introducing a nanoscale pattern in the interacting surfaces. This result is the first experimental demonstration of a technique capable of reducing the Casimir interaction between metallic objects.

\section{Acknowledgments}

The author acknowledges support from the National Science Foundation through Grant PHY-0701636, from Los Alamos National Laboratory through Contract 49423-001-07, and from DARPA through the Casimir Effect Enhancement program. Support from the Nanosacale Imaging Center at IUPUI is also acknowledged. The author is indebted with D. López, V. Aksyuk, S. T. Koev and I.-W. Jung for providing the gratings to perform the measurements. He also recognizes discussions with D. A. R. Dalvit and F. Intravaia.

\section{References}

1. H. B. G. Casimir, Proc. K. Ned. Akad. Wet. 51, 793 (1948).

2. S. K. Lamoreaux, Phys. Rev. Lett. 78, 5 (1997); 81, 5475 (1998).

3. F. Chen, U. Mohideen, G. L. Klimchitskaya and V. M. Mostepanenko, Phys. Rev. A 72, 020101 (2005); ibid 73, 019905 (2006).

4. F. Chen, U. Mohideen, G. L. Klimchitskaya and V. M. Mostepanenko, Phys. Rev. A 74, 022103 (2006).

5. F. Chen, G. L. Klimchitskaya, U. Mohideen and V. M. Mostepanenko, Phys. Rev. Lett. 97, 170402 (2006); Phys. Rev. B 76, 035338 (2007).

6. G. Bressi, G. Carugno, R. Onofrio and G. Ruoso, Phys. Rev. Lett. 88, 041804 (2002). 
7. H. Chan, V. A. Aksyuk, R. N. Kleiman, D. J. Bishop and F. Capasso, Science 291, 1941 (2001).

8. G. Jourdan, A. Lambrecht, F. Comin and J. Chevrier, Europhys. Lett. 85, 31001 (2009).

9. V. B. Svetovoy, P. J. van Zwol, G. Palasantzas and J. Th. M. De Hosson, Phys. Rev. $B$ 77, 035439 (2008).

10. R. S. Decca, E. Fischbach, G. L. Klimchitskaya, D. E. Krause, D. López and V. M. Mostepanenko, Phys. Rev. D 68, 116003 (2003); R. S. Decca, D. López, E. Fischbach, G. L. Klimchitskaya, D. E. Krause and V. M. Mostepanenko, Ann. Phys. (N. Y.) 318, 37 (2005).

11. R. S. Decca, D. López, E. Fischbach, G. L. Klimchitskaya, D. E. Krause and V. M. Mostepanenko, Phys. Rev. D 75, 077101 (2007); Eur. Phys. J. C 51, 963 (2007).

12. M. Boström and B. E. Sernelius, Phys. Rev. Lett. 84, 4757 (2000).

13. V. B. Svetovoy and M. V. Lokhanin, Mod. Phys. Lett. A 15, 1013 (2000); 15, 1437 (2000).

14. C. Genet, A. Lambrecht and S. Reynaud, Phys. Rev. A 62, 012110 (2000); M. Bordag, B. Geyer, G. L. Klimchitskaya and V. M. Mostepanenko, Phys. Rev. Lett. 85, 503 (2000).

15. B. Geyer, G. L. Klimchitskaya and V. M. Mostepanenko, Phys. Rev. A 67, 062102 (2003); V. B. Bezerra, G. L. Klimchitskaya, V. M. Mostepanenko and C. Romero, Phys. Rev. A 69, 022119 (2004).

16. F. Chen, U. Mohideen, G. L. Klimchitskaya and V. M. Mostepanenko, Phys. Rev. A 72, 020101 (2005); 73, 019905 (2006); 74, 022103 (2006); F. Chen, G. L. Klimchitskaya, V. M. Mostepanenko and U. Mohideen, Phys. Rev. B 76, 035338 (2007).

17. M. Popescu and I. D. Simaăndan, J. Phys.: Conf. Ser. 253, 012014 (2010); G. Torricelli, P. J. van Zwol, O. Shpak, C. Binns, G. Palasantzas, B. J. Kooi, V. B. Svetovoy and M. Wuttig, Phys. Rev. A 82, 010101 (2010).

18. J. N. Munday, F. Capasso and V. A. Parsegian, Nature 457, 170 (2009).

19. A. Roy and U. Mohideen, Phys. Rev. Lett. 82, 4380 (1999); F. Chen, U. Mohideen, G. L. Klimchitskaya and V. M. Mostepanenko, Phys. Rev. Lett. 88, 101801 (2002); Phys. Rev. A 66, 032113 (2002).

20. H. B. Chan , Y. Bao, J. Zou J, R. A. Cirelli, F. Klemens, W. M. Mansfield and C. S. Pai, Phys. Rev. Lett. 101, 030401 (2008); Y. Bao, R. Gurout, J. Lussange, A. Lambrecht, R. A. Cirelli, F. Klemens, W. M. Mansfield, C. S. Pai and H. B. Chan, Phys. Rev. Lett. 105, 250402 (2010).

21. H.-C. Chiu, G. L. Klimchitskaya, V. N. Marachevsky, V. M. Mostepanenko and U. Mohideen, Phys. Rev. B 80, 121402 (2009); 81, 115417 (2010).

22. R. S. Decca and D. López, Int. J. Mod. Phys. A 24, 1748 (2009).

23. D. López, R. S. Decca, E. Fischbach and D. E. Krause, Bell Labs Technical Journal 10, 61 (2005). 